\title{
Shedding of cancer susceptibility candidate 4 by the convertases PC7/furin unravels a novel secretory protein implicated in cancer progression
}

Stéphanie Duval ${ }^{1}$, Afnan Abu-Thuraia ${ }^{2}$, Islam E. Elkholi², Rui Chen ${ }^{3}$, Deeptee Seebun ${ }^{3}$, Janice Mayne ${ }^{3}$, Jean-François Côté $\mathbb{D}^{2}$, Daniel Figeys ${ }^{3}$ and Nabil G. Seidah (1) ${ }^{1}$

\begin{abstract}
The proprotein convertases (PCs) are responsible for the maturation of precursor proteins, and are involved in multiple and critical biological processes. Over the past 30 years, the PCs have had great translational applications, but the physiological roles of PC7, the seventh member of the family, are still obscure. Searching for new substrates of PC7, a quantitative proteomics screen for selective enrichment of $\mathrm{N}$-glycosylated polypeptides secreted from hepatic HuH7 cells identified two human type-II transmembrane proteins of unknown function(s): Cancer Susceptibility Candidate 4 (CASC4) and Golgi Phosphoprotein of $130 \mathrm{kDa}$ (GPP130/GOLIM4). Concentrating on CASC4, its mutagenesis characterized the PC7/Furin-shedding site to occur at KR $66 \downarrow$ NS, in HEK293 cells. We defined PC7 and Furin trafficking and activity, and demonstrated that CASC4 shedding occurs in acidic endosomes and/or in the trans-Golgi Network. Our data unraveled a cancer-protective role for CASC4, because siRNA silencing of endogenous CASC4 expression in the invasive triple-negative breast cancer human cell line MDA-MB-231 resulted in a significantly increased cellular migration and invasion. Conversely, MDA-MB-231 cells stably expressing CASC4 exhibited reduced migration and invasion, which can be explained by an increased number of paxillin-positive focal adhesions. This phenotypic cancerprotective role of CASC4 is reversed in cells overexpressing an optimally PC7/Furin-cleaved CASC4 mutant, or upon overexpression of the $\mathrm{N}$-terminally convertase-generated membrane-bound segment. This phenotype was associated with increased formation of podosome-like structures, especially evident in cells overexpressing the N-terminal fragment. In accord, breast cancer patients' data sets show that high CASC4 and PCSK7 expression levels predict a significantly worse prognosis compared to high CASC4 but low PCSK7 levels. In conclusion, CASC4 shedding not only disrupts its anti-migratory/invasive role, but also generates a membrane-bound fragment that drastically modifies the actin cytoskeleton, resulting in an enhanced cellular migration and invasion. This phenotype might be clinically relevant in the prognosis of breast cancer patients.
\end{abstract}

\footnotetext{
Correspondence: Nabil G. Seidah (seidahn@ircm.qc.ca)

'Laboratory of Biochemical Neuroendocrinology, Montreal Clinical Research Institute (IRCM; Affiliated to the University of Montreal), 110 Pine Ave West, Montreal, QC H2W1R7, Canada

${ }^{2}$ Laboratory of Cytoskeletal Organization and Cell Migration, Montreal Clinical Research Institute (IRCM; affiliated to the University of Montreal), 110 Pine Ave West, Montreal, QC H2W1R7, Canada

Full list of author information is available at the end of the article

Edited by S. Tait
}

\begin{abstract}
Introduction
The proprotein convertases (PCs) constitute a family of nine serine secretory proteases that regulate diverse biological processes in both health and disease states ${ }^{1}$. By irreversible proteolysis, PCs are responsible for the activation or inactivation of a variety of precursor proteins, such as growth factors, hormones, receptors and adhesion molecules ${ }^{1}$. Such cleavage or shedding events may also result in the generation of cleaved entities with distinct novel functions. The first seven PCs cleave $(\downarrow)$ precursor
\end{abstract}

\section{(-) The Author(s) 2020}

(c) (i) Open Access This article is licensed under a Creative Commons Attribution 4.0 International License, which permits use, sharing, adaptation, distribution and reproduction cc) in any medium or format, as long as you give appropriate credit to the original author(s) and the source, provide a link to the Creative Commons license, and indicate if changes were made. The images or other third party material in this article are included in the article's Creative Commons license, unless indicated otherwise in a credit line to the material. If material is not included in the article's Creative Commons license and your intended use is not permitted by statutory regulation or exceeds the permitted use, you will need to obtain permission directly from the copyright holder. To view a copy of this license, visit http://creativecommons.org/licenses/by/4.0/. 
proteins at specific single or paired basic amino acid (aa) within the motif $(\mathrm{R} / \mathrm{K})-\left(2 \mathrm{X}_{n}\right)-(\mathrm{R} / \mathrm{K}) \downarrow$, where $\mathrm{X}_{n}=$ $0-3$ spacer $\mathrm{aa}^{2}$. Because of their roles in the processing of many critical secretory substrates, e.g., activation of TGF$\beta^{3}$ and matrix metalloproteases ${ }^{4}$, PCs, such as Furin, PC5, PACE4, and PC7 were implicated in cancer/metastasis ${ }^{5-7}$.

The seventh member of the family (PC7; gene PCSK7) is a ubiquitously expressed protease that often shares substrates with other PCs, especially Furin ${ }^{1,8}$. Recent work by our group identified the type-II transmembrane human transferrin receptor 1 (TfR1) as the first PC7-specific substrate ${ }^{9}$. Even though both Furin and PC7 are enriched in the TGN, Furin cleaves its substrates mostly in the TGN, cell surface or internalized endosomes ${ }^{10,11}$. In contrast, PC7 cleaves its only known specific substrate hTfR1 following its internalization from the cell surface into early endosomes ${ }^{9,12}$. To better understand the PC7 biology and pathophysiology, we undertook an unbiased quantitative proteomics screen of $\mathrm{N}$-glycosylated secreted products from hepatic $\mathrm{HuH} 7$ cells overexpressing PC7. This screen led us to identify two shed type-II transmembrane proteins of unknown biological functions: Cancer Susceptibility Candidate $4(\text { CASC4 })^{13}$, and Golgi Phosphoprotein of $130 \mathrm{kDa}$ (GPP130/GOLIM4) ${ }^{14}$. CASC4 was originally identified in a breast cancer screen in the context of HER $^{+}$overexpression $^{13}$. More recently, CASC4 was also shown to be aberrantly spliced in breast cancer $^{15}$ and glioblastoma ${ }^{16}$, however, the functional consequences of the spliced isoforms were not defined. In addition, a significant increase in secreted (shed) sCASC4 was found upon analysis of the $\mathrm{N}$-glycosylated secretome from highly metastatic breast cancer cell lines ${ }^{17}$.

In this study, we demonstrated that PC7 and Furin specifically shed CASC4 in post-ER acidic compartments, generating an $\mathrm{N}$-terminal membrane-bound domain (NTD) and a secreted C-terminal fragment. Using triplenegative breast cancer MDA-MB-231 cells stably expressing CASC4 or its selected mutants, we demonstrated that wild type (WT) CASC4 enhances cell adhesion by increasing the number of focal adhesions (FA) and actin stress fibers, and that its shedding by PC7/Furin abrogates this phenotype. We then showed that the PC7/ Furin-generated NTD induced the formation of podosome-like structures, implicated in invasion ${ }^{18,19}$. These results provide a novel unique mechanistic rationale for the functions of CASC4 and its shedding by PCs, which impact cellular migration and invasion.

\section{Materials and methods}

Glyco-proteomic analysis of secretome from HEK293 and HuH7 cells overexpressing PC7

\section{Transient transfection}

HEK293 cells (obtained from ATCC) and $\mathrm{HuH7}$ (obtained from the JCRB) were maintained in $5 \% \mathrm{CO}_{2}$ at $37^{\circ} \mathrm{C}$ were seeded at $6 \times 10^{5}$ cells in 6 -well plates and grown to $80 \%$ confluency in Dulbecco's modified Eagle medium (DMEM; Invitrogen) supplemented with $10 \%(\mathrm{v} / \mathrm{v})$ fetal bovine serum (FBS; Invitrogen), $1 \mathrm{~mm}$ sodium pyruvate (Life Technologies) and $28 \mu \mathrm{g} / \mathrm{ml}$ gentamycin (Millipore-Sigma). Cells were transiently transfected with plasmid pIRES2 containing cDNA encoding human PC7 at $2 \mu \mathrm{g}$ and as control pIRES2 empty vector. Transfections were carried out following Lipofectamine 3000 (Invitrogen) recommended protocols with a $1 \mu \mathrm{g}: 1 \mu \mathrm{l}$ ratio of DNA to Lipofectamine reagent in OPTI-MEM media (Invitrogen) for HEK293 cells and 1:4 for $\mathrm{HuH} 7$ cells. Six hours post transfection, media was replaced by $2 \mathrm{ml}$ of fresh OPTI-MEM media. At $24 \mathrm{~h}$ post media change, spent media was collected, centrifuged at $16,000 \times g$ for $2 \mathrm{~min}$ to remove cellular debris and supernatants stored at $-80^{\circ} \mathrm{C}$.

\section{Enrichment of secreted glycoproteome}

Spent media from transient transfections of PC7 and empty vector were concentrated and equilibrated in $8 \mathrm{M}$ urea by ultracentrifugation using Amicon Ultra-15 centrifugal filter units ( $3 \mathrm{kDa}$ cut-off, Millipore-Sigma). A total of $500 \mathrm{ug}$ of proteins were used for glycoprotein enrichment. Proteins were digested with trypsin as described in ref. ${ }^{20}$. Briefly, proteins were reduced with $10 \mathrm{mM}$ dithiothreitol (DTT) at $56^{\circ} \mathrm{C}$ for $45 \mathrm{~min}$, alkylated with $20 \mathrm{mM}$ iodoacetamide (IAA) at room temperature for $1 \mathrm{~h}$ and digested with trypsin at a $1: 50$ ratio at $37^{\circ} \mathrm{C}$ overnight. Glycopeptides were enriched by hydrophilic interaction chromatography solid phase extraction (HILIC-SPE) as described in ref. ${ }^{20}$. Following capture, and washes to remove non-glycosylated peptides, the enriched glycopeptides were eluted from column and dried by vacuum centrifugation. The enriched fraction was deglycosylated using 50 units of PNGaseF (New England Biolabs) in $50 \mu \mathrm{l} 100 \mathrm{mM}$ ammonium bicarbonate at $37^{\circ} \mathrm{C}$ overnight.

\section{LC-MS/MS analysis and database search}

Deglycosylated peptides were analyzed with an HPLCMS/MS as per ref. ${ }^{20}$, using Q Exactive mass spectrometer (ThermoFisher Scientific Inc.) (ThermoFisher). The instrument method consisted of one full MS scan from 300 to $1800 \mathrm{~m} / z$ followed by data-dependent MS/MS scan of the 12 most intense ions, a dynamic exclusion repeat count of 2, and repeat exclusion duration of 30. Data files were processed with MaxQuant (1.2.2.5). The resulting precursor masses were matched to the IPI human database (version 3.68, 87,061 entries), and included the standard MaxQuant contaminant database. Mass tolerances were $6 \mathrm{ppm}$ and $0.05 \mathrm{Da}$ for the precursor and fragment, respectively. Enzyme specificity was set as KR/ $\mathrm{P}$, and a maximum of two missed cleavages was allowed. 
Cysteine residue was set as a static modification of 57.0215 Da, and the methionine oxidation and asparagine deamination were set as a variable modification of 15.9949 and $0.9840 \mathrm{Da}$, respectively. The false discovery rate cutoffs for both peptides and proteins were set at $1 \%$. The protein group file was imported into Perseus (version 1.2.0.17) where identifications from contaminants and reversed databases were removed. Label free quantification was carried out and significant changes in proteins were determined by two-sided $t$-tests.

\section{Plasmids}

Human GPP130 WT (ThermoScientific, Open Bioscience), CASC4-WT (ThermoScientific, Open Bioscience), and its mutants (R60A, R62A, K65A, R66A, AA $65 / 66$, NTD, SP$\triangle \mathrm{TM}$-CASC4, and 5REL) were subcloned, with a V5-tag at the C-terminus into pIRES2-EGFP vector (Clontech). All constructions (human transferrin receptor 1), human Furin, mouse PC5A, mouse PC5B, human PACE4, full-length human PC7, soluble rat PC7, soluble human PC7, and Sar1P(H79G) were cloned in pIRES2-EGFP vector (Clontech).

\section{Cell culture, transfections, and cell treatments}

HEK293 cells were grown in Dulbecco's modified Eagle's medium (DMEM, Invitrogen) with $10 \%$ fetal bovine serum (FBS, Invitrogen), CHO-ldlD and CHO-K1 cells were grown in DMEM/F12 medium with $10 \% \mathrm{FBS}$, MDA-MB-231 cells were grown in Dulbecco's modified Eagle's medium (DMEM, Invitrogen) with $10 \%$ fetal bovine serum (FBS, Invitrogen), MCF10a cells were grown in MEGM Mammary Epithelial Cell Growth Medium BulletKit from Lonza (Catalog \#: CC-3150) $+5 \%$ horse serum. All cells were maintained at $37^{\circ} \mathrm{C}$ under $5 \% \mathrm{CO}$. HEK293 cells were co-transfected with equimolar quantities $(0.5 \mu \mathrm{g})$ of each plasmid using Jetprime Polyplus, CHO-ldlD cells were transfected with equimolar quantities $(1.0 \mu \mathrm{g})$ of each plasmid using FuGene HD, using manufacturer's instructions. MDA-MB-231 cells were transfected using GenJet ${ }^{\mathrm{TM}}$ In Vitro DNA Transfection Reagent for MDA-MB231 Cells (SignaGen Laboratories) with equimolar quantities of plasmids $(1.5-2 \mu \mathrm{g})$ using manufacturer's instructions. At $24 \mathrm{~h}$ post transfection, cells were washed in serum-free medium followed by an additional $20 \mathrm{~h}$ alone or in incubation with $2.5 \mu \mathrm{g} / \mathrm{ml}$ brefeldin A (BFA; Calbiochem), Dynasore ( $80 \mu \mathrm{M}$; Sigma), decanoyl-RVKR-CMK (75 $\mu \mathrm{M}$; Bachem), hexapeptide (DArg $)_{6}(10 \mu \mathrm{M}$; EMD Chemicals), or $20 \mathrm{mM}$ ammonium chloride $\left(\mathrm{NH}_{4} \mathrm{Cl}\right.$; Sigma). For Endo $\mathrm{H}$ and PGNase $\mathrm{F}$ treatments, cell lysates were incubated with endoglycosidase $\mathrm{H}$ (endo $\mathrm{H}$ ) or Peptide- $\mathrm{N}$-Glycosidase F (PGNase F) for $1 \mathrm{~h}$ at $37^{\circ} \mathrm{C}$ (New England Biolabs), cells and media were collected for western blot analysis. All experiments were repeated at last three times and center values represent the mean \pm standard error from the mean.

\section{siRNAs and quantitative RT-qPCR}

A pool of four siRNAs against human CASC4 and a scrambled siRNA (Dharmacon; siGENOME SMARTpool) were transfected with a final $100 \mathrm{nM}$ concentration, using DhamaFECT4 transfection reagent (Dharmacon), using the manufacturer's protocol. Total RNA extraction was performed using $1 \mathrm{ml}$ Trizol reagent (Invitrogen) according to the manufacturer's instructions. Real-time PCR was carried out using Viia7 System (Applied Biosystems). Reactions were run in duplicate for each independent experiment. Human TATA-box binding protein (hTBP) gene was used as an internal control to normalize the variability in expression levels. Supplementary Table S1 summaries oligonucleotide sequences used for human CASC4, human PCSK7 and human Furin. All experiments were repeated at last three times and center values represent the mean \pm standard error from the mean.

\section{Affinity-precipitation of GTP-Rho/Cdc42}

MDA-MB-231 cells were washed with ice-cold Phosphate-buffered saline and lysed in Cytoskeleton Lysis Buffer $\left(50 \mathrm{mM}\right.$ Tris pH 7.5, $10 \mathrm{mM} \mathrm{MgCl}_{2}, 0.5 \mathrm{M} \mathrm{NaCl}$, and $2 \%$ Igepal). Cell lysates were clarified by centrifugation at $10,000 \times g$ at $4{ }^{\circ} \mathrm{C}$ for $1 \mathrm{~min}$, and equal protein concentrations from the different cell lysates were incubated with GST-RBD $(25 \mu \mathrm{g})$ or GST-PAK $(10 \mu \mathrm{g})$ beads at $4{ }^{\circ} \mathrm{C}$ for $60 \mathrm{~min}$. The beads were washed two times with washing buffer $(25 \mathrm{mM}$ Tris $\mathrm{pH} 7.5,30 \mathrm{mM} \mathrm{MgCl}$, $40 \mathrm{mM} \mathrm{NaCl}$ ). Bound Rho/Cdc42 proteins were detected by western blotting using a monoclonal antibody against RhoA/Cdc42 (Cytoskeleton). Densitometry analysis was performed using Image J software (National Institutes of Health). The amount of RBD-bound Rho was normalized to the total amount of Rho/Cdc42 in cell lysates for the comparison of Rho activity (level of GTPbound Rho/ Cdc42) in the different samples. All experiments were repeated at last three times and center values represent the mean \pm standard error from the mean.

\section{Western blot analysis and antibodies}

Cells were lysed in cold Radio-Immunoprecipitation Assay (RIPA) buffer (100 mM Tris- $\mathrm{HCl} \mathrm{pH} \mathrm{8,} 300 \mathrm{mM}$ $\mathrm{NaCl}, 0.2 \%$ SDS, $2 \% \mathrm{NP}-40,1 \% \mathrm{Na}$ deoxycholate) containing protease inhibitors (Roche Applied Bioscience). Proteins were analyzed by SDS-PAGE on $8-12 \%$ TrisGlycine and transferred on a nitrocellulose membrane (GE Healthcare Life Science, No. 10600003) followed by $1 \mathrm{~h}$ blocking in Li-Cor blocking buffer (Li-Cor) or in 5\% milk in TBST-T. Membranes were then incubated with primary antibody overnight. Proteins were visualized using mouse anti-V5 (1/2000, Invitrogen), rabbit anti-PC7 (1:10,000, homemade or 1:5000 Cell Signaling Technologies), Furin (1:5000, Invitrogen), rabbit anti $\beta$-actin (1:5000, SigmaAldrich), anti-Tubulin (1:5000, Sigma-Aldrich), p-paxillin 
(Y118) (ThermoFisher), paxillin (Transduction Laboratories), CASC4 (1:500, Abcam), Cdc42 (1:250, Cytoskeleton), RhoA (1:500; Cytoskeleton), or a horseradish peroxidase (HRP)-conjugated mAb V5 (1:10,000; SigmaAldrich), or anti-Flag M2 HRP (1:3000; Sigma-Aldrich). Bound primary antibodies were detected with corresponding species-specific fluorescent anti-mouse antibody 680 (Mandel) (1:10,000) or anti-rabbit Ab 800 (Mandel), and revealed using LiCor Bioscience, or with corresponding species-specific HRP-labeled secondary antibodies (1:10,000; Invitrogen) and revealed by enhanced chemiluminescence (ECL; Amersham). Quantifications were done using Image Studio Lite v.4.0 and Image J software (National Institutes of Health). All experiments were repeated at last three times and center values represent the mean \pm standard error from the mean.

\section{Boyden migration and invasion assays}

For migration assays, $1 \times 10^{5}$ cells were seeded into a transwell $(6.5 \mathrm{~mm}$, Polycarbonate membrane $0.8 \mu \mathrm{M}$, VWR) and allowed to migrate towards 10\% FBS DMEM medium into the lower chamber as a chemoattractant during $6 \mathrm{~h}$. For invasion, $5 \times 10^{4}$ cells were seeded into a Matrigel matrix (Corning) and allowed to invade during $16 \mathrm{~h}$ towards $10 \%$ FBS DMEM medium into the lower chamber. After migration/invasion, cells were aspirated from the transwell and the membranes were washed before fixation by $4 \%$ paraformaldehyde (ThermoScientific) for $10 \mathrm{~min}$. Following fixation, membranes were washed three times and mounted onto a microscope slide (Fisher Scientific) with ProLong Gold antifade with DAPI (Invitrogen) to stain for nucleus. The fixed membranes were analyzed on a DMRB microscope at $\times 20$ amplification, ten pictures were taken per condition and nuclei counted. All experiments were repeated at last three times and center values represent the mean \pm standard error from the mean.

\section{Wound healing assay}

Confluent monolayer of cells was scratched with a $200 \mu \mathrm{l}$-pipette tip to generate a scratch wound. To evaluate the distance traveled by the cells, pictures were taken with a Leica microscope at 6 and $12 \mathrm{~h}$ post-scratch. Images were analyzed using Image J software (National Institutes of Health). All experiments were repeated at last three times and center values represent the mean \pm standard error from the mean.

\section{Microscopy analysis and antibodies}

For F-actin staining, cells were seeded on Fibronectin (Sigma) coated $(20 \mu \mathrm{g} / \mathrm{ml})$ glass coverslip into 24 well plates and grown for $48 \mathrm{~h}$. Cells were then fixed with warm paraformaldehyde (4\%) for 20 min and permeabilized with PBS $1 x+$ Triton $0.1 \%$. Followed permeabilization, cells were stained for primary antibodies: paxillin (Transduction laboratories), anti-LDLR (R\&D systems), anti-Golgin97 (Santa Cruz Biotechnology), V5 (Invitrogen), and TKS5 (Millipore-Sigma) for $1 \mathrm{~h}$ followed by fluorescent corresponding secondary antibodies or fluorescent coupled 555-Phalloidin to stain for F-actin for $30 \mathrm{~min}$. Coverslips were then mounted on microscope slide (Fisher Scientific) with ProLong Gold antifade with DAPI (Invitrogen) to stain for nucleus. Colocalization of fluorescently labeled protein was quantified with IMARIS analysis software (8.2.1) along with aXTension script named Colocalize Spots. We used the same approach as mentioned in Rajan et $\mathrm{al}^{21}$. Positive signals were found using the Imaris function spots from each fluorescent marker images. The spot diameter used was $1.2 \mu \mathrm{m}$ with the same quality factor for each image. The Colocalize Spots script considers colocalization between two spots when their center to center distance is equal of inferior to $0.8 \mu \mathrm{m}$. FA quantifications were done using Image $J$ software (National Institutes of Health) with a program previously described $^{22}$. All experiments were repeated at last three times and center values represent the mean \pm standard error from the mean.

\section{Clinical data analysis}

METABRIC $^{23,24}$ and TCGA $^{25}$ data sets were accessed through the cBioPortal online platform ${ }^{26,27}$. To investigate the correlation between the CASC4 and PCSK7 mRNA levels, the plot tool was used. To correlate the association of CASC4 and PCSK7 mRNA expression levels with the survival rate in the METABRIC data set, the expression level values of both genes (in $z$-scores) were downloaded for the whole data set. Threshold of $z$-score $=/$ higher than 1 is used to identify patients with high expression of PCSK7 or CASC4 and threshold of $z$-score $=$ /lower than -1 is used to identify patients with low expression of PCSK7.

\section{Results}

Mass spectrometry identifies two novel type-II transmembrane proteins cleaved by $\mathrm{PC7}$

To identify novel PC7-specific substrates, we used a mass spectrometry approach that analyses affinity purified $\mathrm{N}$-glycoproteins secreted in the media (Fig. 1a). Analysis of secreted $\mathrm{N}$-glycosylated products by this procedure avoids the limitations of the low concentrations of proteins of interest, high abundance of non-glycosylated plasma proteins in the incubation medium, or contamination by cytosolic proteins released from broken cells ${ }^{28}$. Indeed, we selectively enriched samples for $\mathrm{N}$-glycosylated peptides using hydrophilic interaction chromatography solid phase micro-extraction (HILIC-SPE), before analyzing them by HPLC-ESI-MSMS (Fig. 1a). Accordingly, we compared the quantitative changes of 645 and 867 enriched glycosylated 


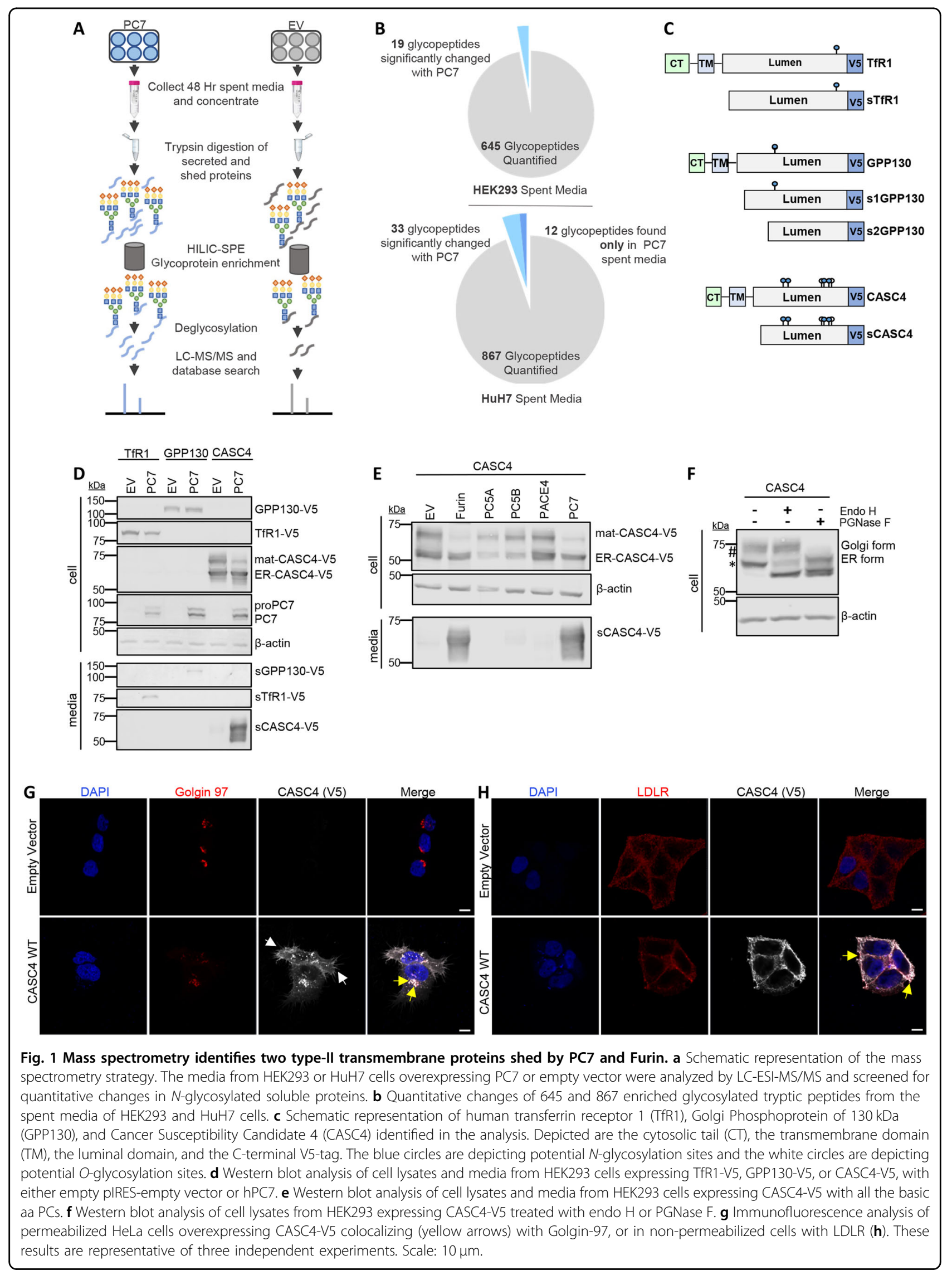


tryptic peptides from the spent media of human embryonic kidney cells (HEK293) and human hepatic (HuH7) cells, both endogenously expressing TfR1, overexpressing either human PC7 or an empty vector (EV) control, respectively (Fig. 1b, and raw data in Supplementary Tables S2 and S3). From HEK293 spent media with PC7, 19 glycopeptides had significantly enhanced levels, 18 of which exhibited potential PC-cleavage sites (K/R)- $\left(2 \mathrm{X}_{n}\right)$ $(\mathrm{K} / \mathrm{R}) \downarrow$ (Supplementary Table S2) ${ }^{1}$. From the HuH7 spent media with PC7, 33 glycopeptides had significantly enhanced levels and an additional 12 glycopeptides were only observed with PC7 expression (Supplementary Table S3). Of these, only ten parent glycoproteins exhibited potential PC-cleavage sites. These results allowed us to confirm previously known PC7 substrates, such as $\operatorname{ADAM} 17^{29}$, Sortilin ${ }^{8}$, and human $\mathrm{TfR} 1^{9}$, as well the identification of two novel substrates, the type-II transmembrane proteins CASC4 and GPP130/GOLIM4 (Supplementary Tables S2, S3 and Fig. 1c).

In order to confirm that CASC4 and GPP130 were cleaved by PC7, we co-expressed in HEK293 cells cDNAs coding for V5-tagged human TfR1, CASC4 or GPP130 with those encoding human PC7 or an empty vector control (Fig. 1d). Compared to control conditions, western blot (WB) analyses revealed the presence of secreted products (sCASC4 and sGPP130, or as control sTFR1) from cells co-expressing $\mathrm{PC} 7$, confirming that the membrane-bound CASC4 and GPP130 are shed by PC7, similarly to TfR1. In view of the connection of CASC4 to cancer (see later), in this study, we concentrated on the consequences of the shedding and functional modulation of CASC4 by PCs in details.

\section{CASC4 is shed by PC7 and Furin}

To assess whether CASC4 shedding is specific to PC7, in HEK293 cells we co-expressed CASC4 with all the basic amino acid specific PCs (Furin, PC5A, PC5B, PACE4, and PC7 $)^{1}$, or with an empty vector control. WB analysis revealed the presence of a fragment released only in the media of cells expressing PC7 or Furin, but not from cells overexpressing the other PCs (Fig. 1e). Interestingly, the molecular sizes of the PC7 and Furin-shed products were similar (Fig. 1e), suggesting that they cleave CASC4 at, or close to, the same site. To further characterize the difference between the observed $\sim 66$ and $\sim 75 \mathrm{kDa}$ CASC4 cellular proteins (Fig. 1d, e), we treated the cell lysates with endoglycosidase $\mathrm{H}$ (endo $\mathrm{H}$ ) that cleaves immature, mannose-rich sugars, or with PGNase $\mathrm{F}$, which cleaves both immature and mature $\mathrm{N}$-linked oligosaccharides (Fig. 1f). The data demonstrated that the $\sim 66 \mathrm{kDa}$ CASC4 is likely to reside in the endoplasmic reticulum (ER), since its apparent molecular size is reduced to $\sim 61 \mathrm{kDa}$ ("; ER-CASC4) when treated with endo $\mathrm{H}$. We also noticed the presence of a residual amount of $\sim 66 \mathrm{kDa}$ CASC4 after endo $\mathrm{H}$ digestion, suggesting the presence of an $\mathrm{O}$-glycosylated form that is not $N$-glycosylated. Indeed, the mature $\sim 75 \mathrm{kDa} C A S C 4$ protein (\#; mat-CASC4) is insensitive to endo $\mathrm{H}$, suggesting it has exited the ER, but its size is reduced to both $\sim 61$ and $\sim 66 \mathrm{kDa}$ upon PGNase F digestion. As expected from the zymogen activation of Furin in the trans-Golgi network (TGN) ${ }^{11,30}$ and that of PC7 occurring in early endosomes ${ }^{9,12}$, only the $\sim 75 \mathrm{kDa}$ mat-CASC4 is processed by either PC7 or Furin (Fig. 1d, e). In support of these data, we compared the migration pattern of CASC4 expressed in CHO-K1 cells to that in CHO-ldlD cells, which are deficient in UDP- $N$-acetylgalactosamine and hence lack $O$-glycosylation activity ${ }^{31}$. The data clearly show that $O$-glycosylation adds $\sim 11 \mathrm{kDa}$ to the apparent molecular size of mature CASC4 (Supplementary Fig. S1).

Immunofluorescence staining of HeLa cells revealed that CASC4 co-localizes with the TGN marker Golgin-97 (yellow arrows, Fig. 1g). When cells were stained under non-permeabilized conditions, CASC4 is only detected at the cell surface, colocalizing with the cell-surface marker low-density-lipoprotein-receptor (LDLR) (yellow arrows, Fig. 1h). Interestingly, we observed a change in cell morphology upon CASC4 expression, providing a clue to elucidate a novel biological function of CASC4.

\section{CASC4 cleavage by PC7 and Furin occurs at Arg66 $\downarrow$}

Because PC7 and Furin cleave substrates after single or paired basic amino acids $(\mathrm{aa})^{1}$, we mutated the basic aa in the proposed P1-P2 ${ }^{31}$ dibasic motif $\mathbf{K R}_{66} \downarrow$, as well as the putative P5 $\mathbf{R}_{60}$ and P7 $\mathbf{R}_{62}$ sites into alanine (Fig. 2a, b). WB analyses of HEK293 cells co-expressing PC7 or Furin with WT-CASC4 or its Ala-mutants R60A, R62A, K65A or R66A, demonstrated that the $\mathrm{K} 65 \mathrm{~A}$ and especially R66A variants are resistant to shedding by $\mathrm{PC} 7$ and Furin (Fig. 2b), while the other mutants did not significantly affect shedding. To further emphasize that $\mathbf{K R}_{\mathbf{6 6}} \downarrow$ is the shedding site, we generated CASC4 mutant proteins harboring an optimized PC-site RRRRR $\mathbf{6 6}_{\mathbf{6}} \mathrm{EL}^{1,32}$ (called 5REL), or a PC-non-cleavable $\mathrm{AA}_{65 / 66}$ mutant. Accordingly, in HEK293 cells, processing of the CASC4-AA $65 / 66$ mutant protein was impaired compared to WT (Fig. 2c, d). Expression of the 5REL mutant generated a soluble fragment, even when overexpressed with an empty vector control (Fig. 2c, d), with an apparent molecular size similar to that of the PC7/Furin-cleaved form of WTCASC4, supporting that $\mathbf{K R}_{66} \downarrow$ is the shedding site. To demonstrate that the heterogeneity of the secreted forms of CASC4 is due to O-glycosylation (Fig. 2c), we coexpressed CASC4-WT and its mutants 5REL and $\mathrm{AA}_{65 / 66}$ in $\mathrm{CHO}$-ldlD cells that cannot $O$-glycosylate proteins ${ }^{33}$. As expected, the secreted sCASC4 now migrates as a sharp protein band $(\sim 61 \mathrm{kDa})$, confirming the $O$-glycosylation of CASC4 (Fig. 2e). Taken together, these data 

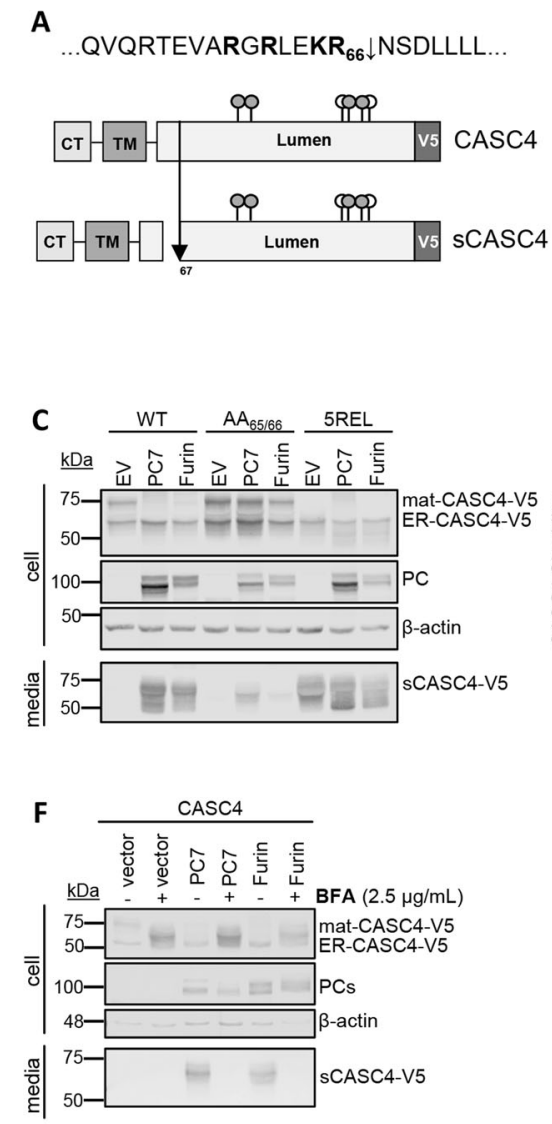
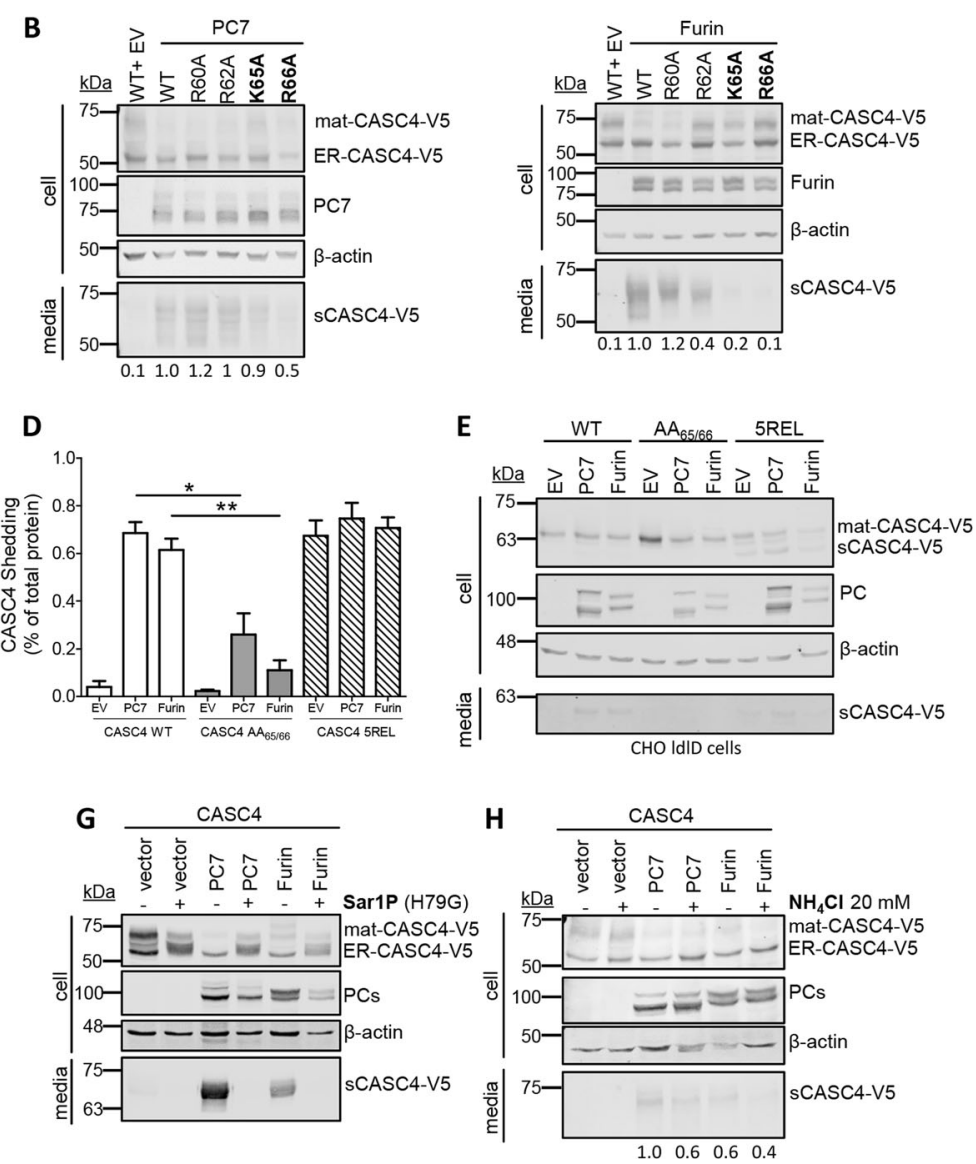

Fig. 2 CASC4 cleavage by PC7 and Furin occurs after Arg66 $\downarrow$ in acidic compartment. a Schematic representation of the predicted cleavage site $K_{6} \downarrow$ generating a $\mathrm{N}$-terminal fragment and a luminal domain. The gray circles are depicting potential $\mathrm{N}$-glycosylation sites and the white circles are depicting O-glycosylation sites. b Western blot analysis of cell lysates and media from HEK293 cells overexpressing CASC4-V5 WT and different point mutation (R60A, R62A, K65A, or R66A) co-expressed with either plRES-empty vector or with hPC7 or hFurin. c, d Western blot analysis and quantifications of cell lysates and media from HEK293 cells overexpressing CASC4-WT-V5, CASC4-5REL-V5 optimally cleaved mutant, or a PC-noncleavable $\mathrm{AA}_{65 / 66}$ site, co-expressed with either pIRES-empty vector or with hPC7 or hFurin. e Western blot analysis of cell lysates and media from CHO-IdID cells overexpressing CASC4-V5 WT, CASC4-5REL optimally cleaved mutant or a PC-non-cleavable AA $65 / 66$ site, co-expressed with either pIRES-empty vector or with hPC7 or hFurin. $\mathbf{f}$ Western blot analysis of cells lysates and media from HEK293 cells overexpressing CASC4-V5 WT, pIRESempty vector, and $\mathrm{hPC7}$ or hFurin treated with Brefeldin $\mathrm{A}(2.5 \mu \mathrm{g} / \mathrm{ml})$ or $\mathrm{NH}_{4} \mathrm{Cl}(20 \mathrm{mM})(\mathbf{g})$. $\mathbf{h}$ Western blot analysis of cell lysates and media from HEK293 cells overexpressing CASC4-V5 WT, pIRES-empty vector, dominant-negative Sar1P-(H79G), and/or with hPC7 or hFurin. These results are representative of three independent experiments. Error bars indicate averaged values \pm standard error from the mean (SEM). $P$-values: ${ }^{*} P \leq 0.05$, **P $<0.01$, (two-sided Student's t-test).

confirm that CASC4 cleavage occurs at $\mathbf{K R}_{66} \downarrow N S$, which generates a secreted luminal domain and a short $\mathrm{N}$ terminal domain (NTD) composed of the cytosolic tail and transmembrane domain.

\section{Shedding occurs in acidic compartments of the secretory pathway}

Brefeldin A abrogated CASC4 shedding by $\mathrm{PC} 7$ and Furin (Fig. 2f). Since Brefeldin A blocks transport of secretory proteins out from the $\mathrm{ER}^{34}$, this suggests that shedding occurs in a post-ER compartment. The same conclusion was reached upon expression of the dominantnegative Sar1p-(H79G) (Fig. 2g), a GTP-restricted mutant that blocks COP-II vesicle formation ${ }^{35}$. Hence, proper COP-II vesicle formation and trafficking from the ER to the Golgi is necessary for PC7 and Furin to shed CASC4. In addition, we used the cell non-permeable general $\mathrm{PC}$ inhibitor D6R, which inhibits PC activity at the cell surface, in comparison to decanoyl-RVKR-chloromethylketone, a cell-permeable PC inhibitor ${ }^{9}$. Our results show that CASC4 cleavage by both $\mathrm{PC} 7$ and Furin is reduced by dec-RVKR-CMK, suggesting that the cleavage occurs intracellularly (Supplementary Fig. S2). On the other hand, both D6R and Dynasore (an inhibitor of endocytic vesicles $^{9}$ ) treatments do not inhibit CASC4 shedding, strongly suggesting that shedding occurs intracellularly 


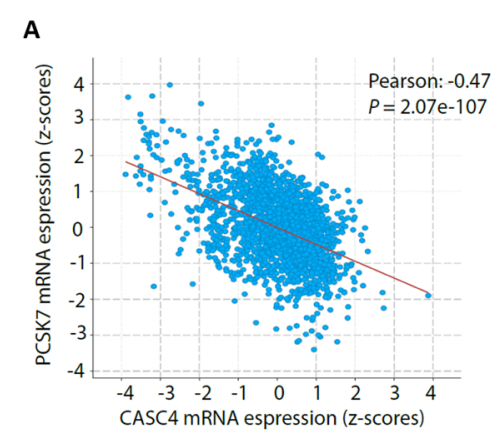

B
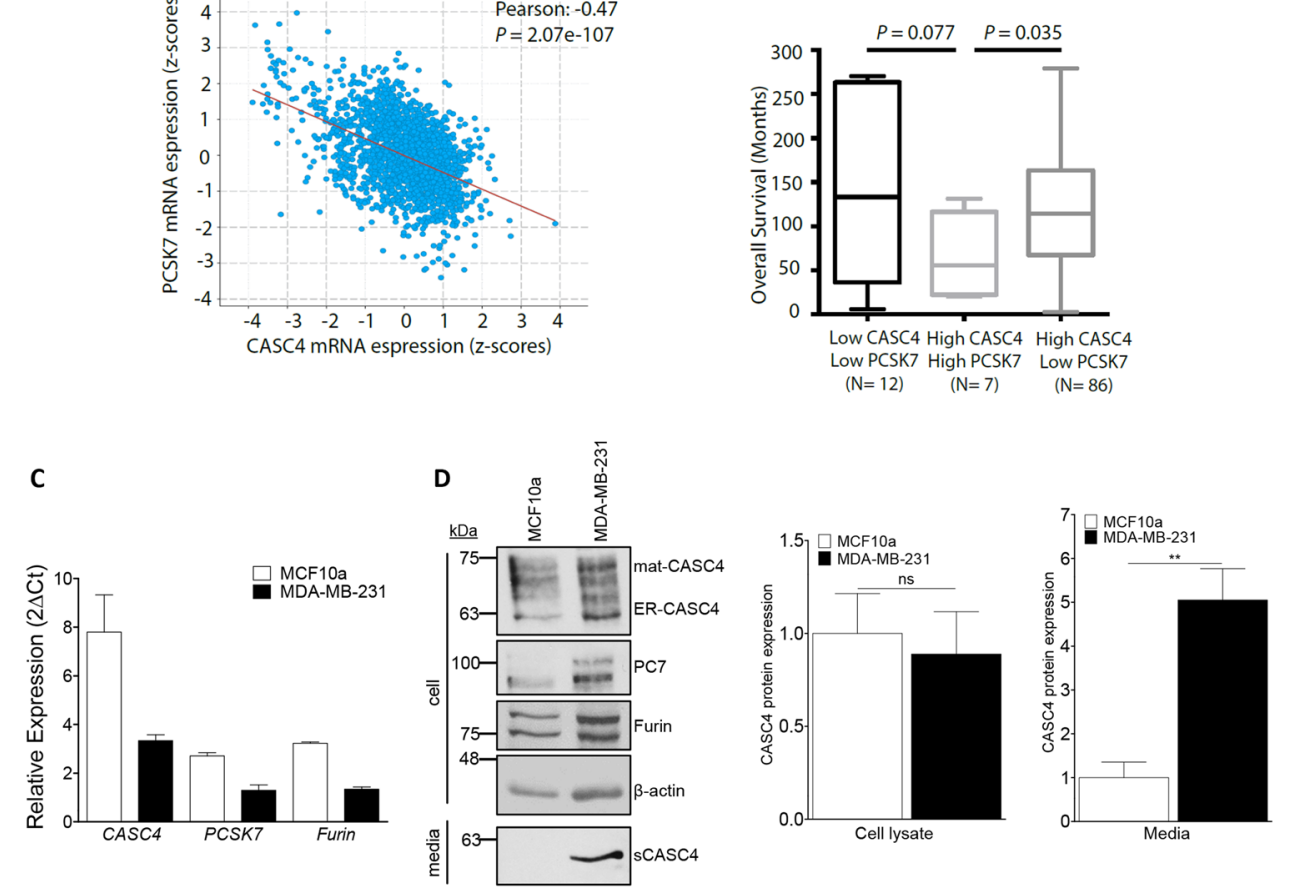

Fig. 3 CASC4 association with PC7 predicts poor prognosis in breast cancer patients. a Correlation of CASC4 and PCSK7 mRNA expression levels (in z-scores) in the METABRIC patients' data set. $\mathbf{b}$ Association of the expression levels of CASC4 and PCSK7 genes with the survival patients' rate. c qPCR analysis of hCASC4, hPCSK7, and hFurin in MCF10a and MDA-MB-231 cells. d Western blot analysis and quantifications of cell lysates from MCF10a or MDA-MB-231 cells. $P$-values: ${ }^{*} P<0.01$, n.s. not significant (two-sided Student's $t$-test). All experiments were repeated at last three times and center values represent the mean \pm standard error from the mean.

and does not require endocytosis from the plasma membrane (Supplementary Fig. S2). Taken together these results combined with our observations of a reduced shedding upon treatment of cells with the alkalinizing agent ammonium chloride $\left(\mathrm{NH}_{4} \mathrm{Cl}\right)$ to block the acidification of intracellular compartments (Fig. 2h), led us to conclude that shedding occurs in an acidic compartment such as TGN or endosomes originating from the TGN, where enzymatic activity was previously described for both PC7 and Furin ${ }^{9,12,31}$.

CASC4 is expressed in metastatic breast cancer cells and its association with PC7 predicts poor prognosis in breast cancer patients

CASC4 is an uncharacterized protein reported to be associated with a potentially bad prognosis in breast cancer $^{13}$, and its gene is aberrantly spliced in breast cancer cells ${ }^{15}$. In addition, metastatic MDA-MB-453 triplenegative breast cancer cells exhibit increased levels of secreted sCASC4 compared to non-cancerous breast cell lines ${ }^{17}$. This motivated us to investigate the potential biological role of the PC7-CASC4 association in the context of breast cancer. First, we interrogated the METABRIC clinical data set, the largest breast cancer data set encompassing genomic data from $\sim 2500$ samples. Although, CASC4 mRNA levels showed a significant negative correlation with those of PCSK7 (Fig. 3a), the expression levels of the latter could differentiate the survival rate of the patients with high CASC4 levels (Fig. 3b). Indeed, patients with high CASC4 and high PCSK7 expression levels had a significantly worse prognosis that those with high CASC4 but low PCSK7 mRNA levels (Fig. 3b).

Second, we analyzed the expression levels of CASC4, PCSK7, and Furin mRNA levels in MCF10a (human noncancerous breast epithelial cell line) versus MDA-MB-231 (highly metastatic triple-negative breast cancer cell line) cells by $\mathrm{qPCR}$. The three genes are $\sim 2-2.5$-fold more expressed in MCF10a versus MDA-MB-231 (Fig. 3c). However, at the protein level, WB analysis revealed that while CASC4 is similarly expressed in both cell lines, its shedding into the media was mostly observed in MDAMB-231 cells (Fig. 3d). In addition, the protein expression levels of PC7 and Furin were similar in both cells (Fig. 3d). We suggest that the TGN localization of CASC4 (Fig. 1g) and that of the active form of its processing enzymes ${ }^{12}$ may provide a favorable environment to allow its shedding in MDA-MB-231 cells compared to MCF10a cells. In 
A

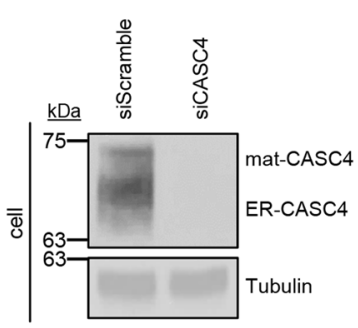

B
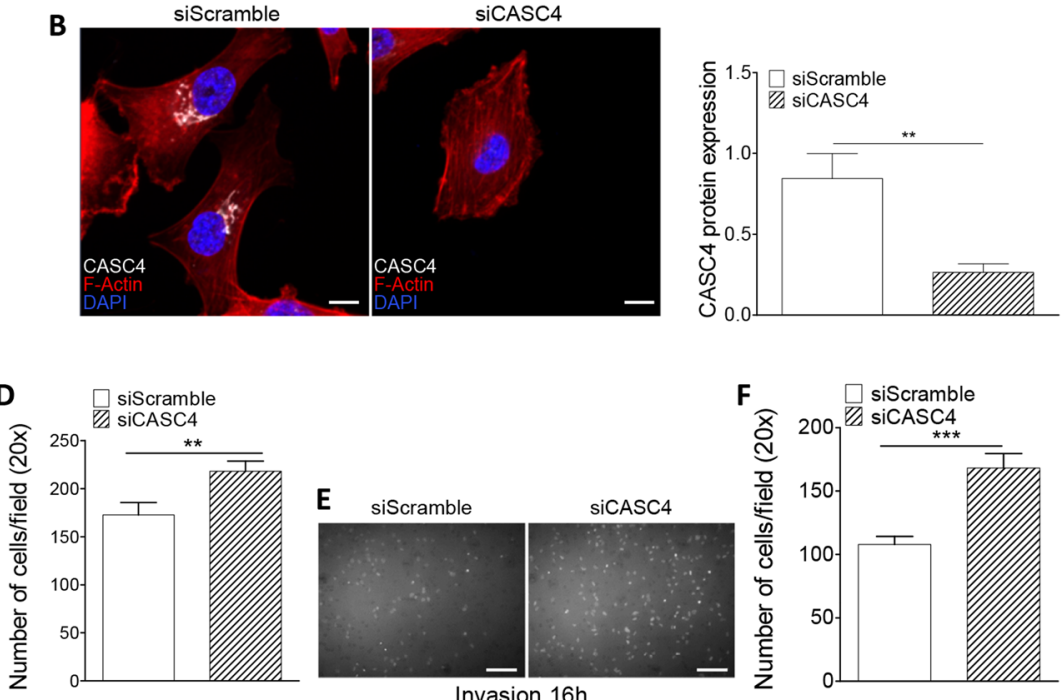

Invasion $16 \mathrm{~h}$

Fig. 4 CASC4 knockdown increases migration and invasion in MDA-MB-231 metastatic breast cancer cells. a Western blot analysis and quantification of cell lysates from MDA-MB-231 cells after $48 \mathrm{~h}$ siRNA knockdown of endogenous CASC4. $\mathbf{b}$ Immunofluorescence analysis of MDA-MB231 cells after $48 \mathrm{~h}$ siRNA knockdown of endogenous CASC4 stained for CASC4 (white labeling), phalloidin (red labeling) and nucleus stained with DAPI (blue labeling). The ability of MDA-MB-231 cells, after $48 \mathrm{~h}$ siRNA knockdown of endogenous CASC4 to migrate ( $6 \mathrm{~h})(\mathbf{c}, \mathbf{d})$ or invade (16 h) (e, $\mathbf{f})$ was assessed by counting the number of cells stained with DAPI on the underside of a polycarbonate membrane under a phase contrast microscope $(\times 20)$. These results are representative of at least three independent experiments. Error bars indicate averaged values \pm standard error from the mean (SEM). $P$-values: ${ }^{* *} P<0.01,{ }^{* * *} P \leq 0.001$ (two-sided Student's $t$-test). Scale: $10 \mu \mathrm{m}$.

addition, we also assessed by qPCR the expression levels in these cells of the endogenous inhibitors of Furin and PC7, namely PAR $1^{36}$, GBP2, and GBP5 ${ }^{37}$. The data show that PAR1 is expressed at $\sim 6$-fold higher levels in MCF10a than in MDA-MB-231 cells, whereas GBP2 is unchanged in both cell lines and GBP5 is barely expressed (Supplementary Fig. S3). Thus, the low expression of the Furin/ PC7 inhibitor PAR $1^{36}$ in MDA-MB-231 cells rationalizes the higher activity of Furin and PC7 therein. Altogether these data suggest that the PC7-CASC4 association and specifically the PC7-mediated shedding of CASC4 might have functional consequences in terms of breast cancer aggressiveness (i.e., metastasis).

\section{CASC4 modulates cell migration and invasion}

The cytoskeletal extensions observed upon overexpression of CASC4 in Hela cells (Fig. 1g), and the correlation between PCSK7-CASC4 mRNA levels with breast cancer patients' survival (Fig. 3a, b) suggested that CASC4 may impact cell migration and invasion, two essential steps of the metastatic process. We thus investigated whether siRNA knockdown of CASC4 alters cell migration and invasion in MDA-MB-231 cells. The siRNA-induced silencing was efficient since it reduced by $\geq 80 \%$ endogenous CASC4 protein, as observed by WB (Fig. 4a) and immunofluorescence (Fig. 4b). Depletion of CASC4 resulted in a significant increase in cell migration $(+30 \%)$ and invasion $(+60 \%)$ as assessed by Boyden
Migration and Invasion Assays, respectively (Fig. 4c-f). These results suggest that expression of CASC4 in MDAMB-231 cells reduces their cell migration and invasion potential.

Accordingly, we generated MDA-MB-231 cells stably expressing CASC4-WT, CASC4-5REL (with the constitutively PC-cleaved RRRRR ${ }_{66} \downarrow$ EL motif), or an empty vector control (Fig. 5a). We confirmed the protein expression in these cells by WB analysis (Fig. 5b). Since the lack of CASC4 expression resulted in enhanced migration and invasion (Fig. 4c-f), we evaluated the migration potential of the stable cell lines first by using a wound healing assay. Compared to control or cells expressing CASC4-5REL, only expression of CASC4-WT resulted in significant inhibition of wound closure postscratching, especially evident after $12 \mathrm{~h}$ (Fig. 5c, d). In complementary Boyden Migration and Invasion Assays (Fig. 5e-h), CASC4-WT overexpression significantly reduced cell migration $(-50 \%)$ and invasion $(-70 \%)$, supporting a protective role of CASC4 as a negative regulator of cellular movement. Although overexpression of CASC4-5REL did not show a significant migration phenotype (Fig. 5e, f), its effect on invasion was intermediate between WT and control (Fig. 5g, h). Taken together, these data suggest that CASC4 represses cellular migration, possibly by acting on key players orchestrating the cellular architecture, and that CASC4 shedding by PCs largely prevents this effect. 
A
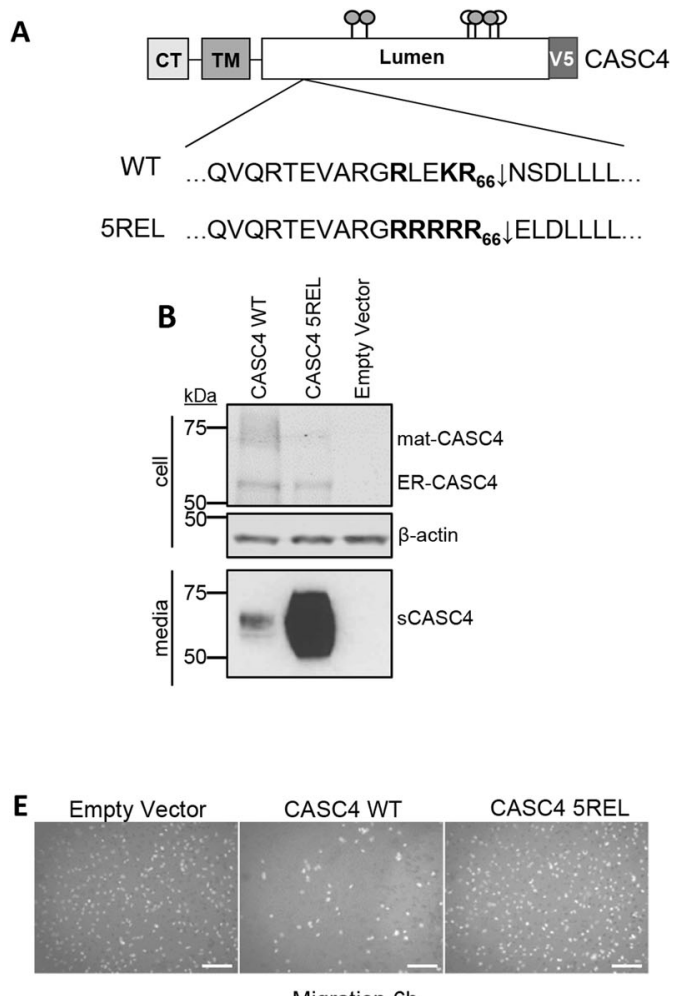

Migration 6h

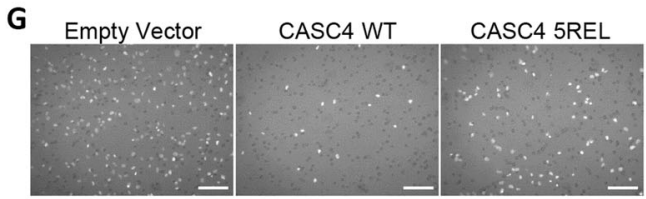

Invasion $16 \mathrm{~h}$

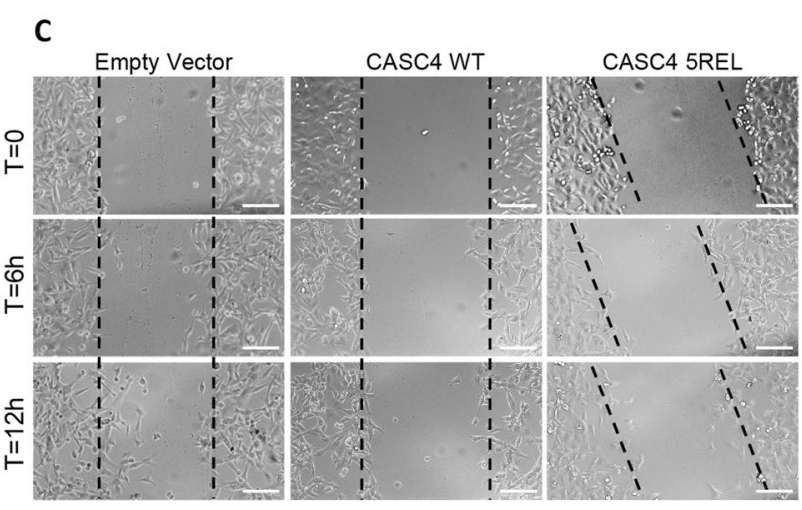

D
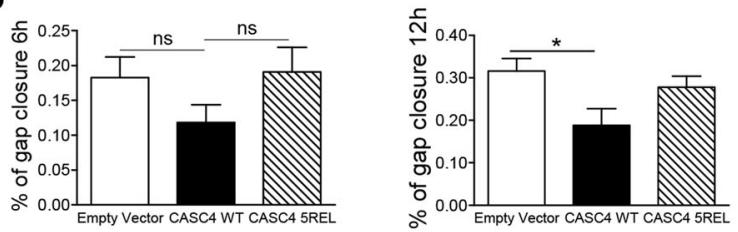

$\mathbf{F}$

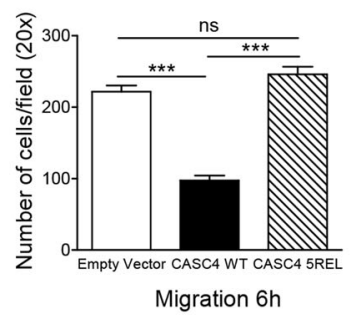

Fig. 5 CASC4 overexpression decreases cell migration and invasion. a Schematic representation of CASC4-WT and amino acid mutations used for the generation MDA-MB-231 stable cells. b Western blot analysis of lysates from MDA-MB-231 stable cells expressing pIRES-empty vector, CASC4WT-V5, or CASC4-5REL-V5. c, d Wound healing assay images and quantifications after $6 \mathrm{~h}, 12 \mathrm{~h}$ post-cell monolayer scratching in MDA-MB-231 stable cell lines. $\mathbf{e}-\mathbf{h}$ The ability of MDA-MB-231 stable cells to migrate $(6 \mathbf{h})(\mathbf{e}-\mathbf{f})$ or invade $(16 \mathbf{h})(\mathbf{g}-\mathbf{h})$ was assessed by counting the number of cells stained with DAPI on the underside of a polycarbonate membrane under a phase contrast microscope $(\times 20)$. These results are representative of at least three independent experiments. Error bars indicate averaged values \pm standard error from the mean (SEM). $P$-values: ${ }^{*} P \leq 0.05$, ${ }^{* * *} P \leq 0.001$, n.S. not significant (two-sided Student's t-test).

\section{CASC4 enhances the number of FA formation and impairs} Cdc42 activation

To investigate how CASC4 interferes with cellular migration/invasion, we analyzed the actin architecture and FAs by immunofluorescence for phalloidin- and paxillin staining, respectively. Interestingly, CASC4-WT cells exhibited a higher number and a trend for larger paxillinpositive FA complexes (Fig. 6a, b), as well as $\sim 3$-fold more $\mathrm{Tyr}_{118}$-phosphorylated paxillin (Fig. 6c). In addition, the overall architecture of CASC4-WT cells was severely impaired, as evidenced by the induction of actin stress fibers at the expense of cortical actin (Fig. 6a). This architectural cellular phenotype could explain the observed reduced migration observed in CASC4-WT cells (Fig. 5c-h). The actin architecture in cells expressing
CASC4-5REL, while also severely disrupted, exhibited a very different phenotype with a completely disorganized paxillin staining (Fig. 6a).

We next characterized in our stable cell lines the activation/inactivation of Rho GTPases, which are molecular switches that control actin cytoskeleton and FA dynamics $^{38,39}$. The levels of the active forms of the Rho GTPases Cdc42 and RhoA implicated in FA turnover ${ }^{40,41}$ were quantified by specific GST pull-downs. The data revealed a significant decrease in the levels of active Cdc42 in cells overexpressing CASC4-WT, compared to empty vector and CASC4-5REL, whereas those of active RhoA remained unchanged (Fig. 6d). This suggests that CASC4-WT, but not CASC4-5REL, blunts the activation of Cdc42, resulting in increased assembly of FA and stress fibers (Fig. 6a). 

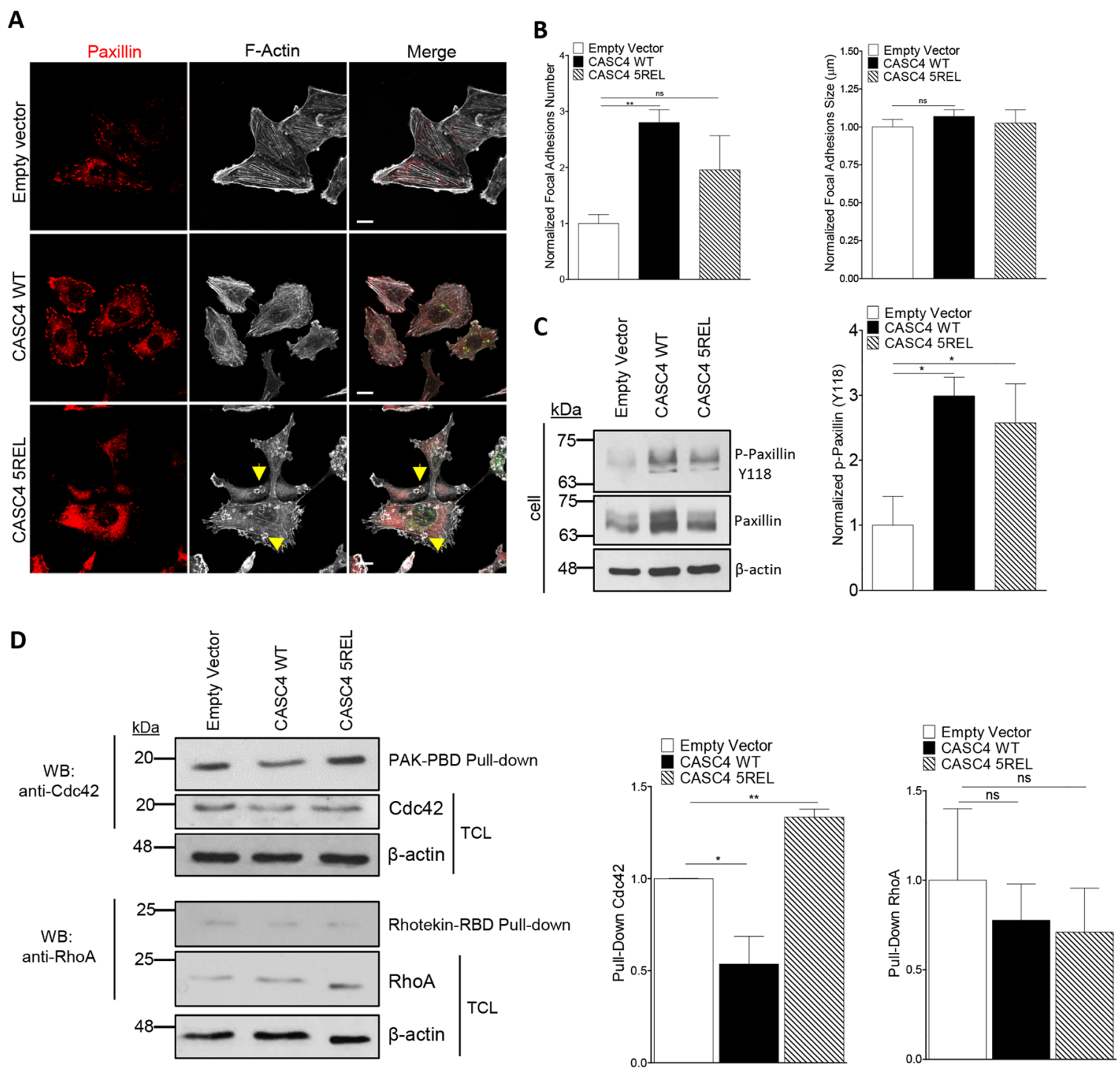

Fig. 6 CASC4 enhances focal adhesions and perturb actin architecture. a Immunofluorescence analysis of stable MDA-MB-231 cell lines expressing pIRES-empty vector, CASC4-WT or CASC4-5REL stained for focal adhesion marker (Paxillin; red labeling), phalloidin (F-actin; white labeling), and V5 (green labeling). Yellow arrows are highlighting round actin circles generated in cells expressing CASC4-5REL. b Quantifications of focal adhesions (Paxillin-positive areas) number and size. $\mathbf{c}$ Western blot analysis and quantifications of cell lysates from MDA-MB-231 stable cells showing endogenous paxillin and p-paxillin (Y118). $\mathbf{d}$ Western blot analysis and quantifications of cells lysates from MDA-MB-231 stable cells incubated with the indicated GST-fusion proteins bound to glutathione beads. The precipitated proteins were detected by immunoblotting with anti- $C \mathrm{cdc} 42$ or antiRhoA antibodies. TCL total cell lysate. These results are representative of at least three independent experiments. Error bars indicate averaged values \pm standard error from the mean (SEM). $P$-values: ${ }^{*} P \leq 0.05{ }^{* *} P<0.01$, n.s. not significant (two-sided Student's $t$-test). Scale: $10 \mu m$.

\section{CASC4 NTD impairs actin organization and induces invadopodia-like structures}

Notably, cells expressing CASC4-5REL presented round actin rings (depicted with yellow arrows) (Fig. 6a). These structures are reminiscent of circular dorsal ruffles, actin structures present on the dorsal surface of cells in response to stimuli (i.e., EGF, PDGF) ${ }^{42}$, and/or invadosomes (invadopodias/podosomes) structures implicated in actin remodeling and invasion in both cancerous (invadopodias) and non-cancerous cells (podosomes) ${ }^{18,19}$. Since colocalization of F-actin with protein tyrosine kinase substrate 5 (Tks5) and extracellular matrix degradation can define invadosomes ${ }^{19}$, we characterized these structures by immunofluorescence staining for F-actin and Tks5. The data showed that Tks5 staining co-localizes (yellow arrows) with the F-actin structures in CASC4-5REL cells (Fig. 7a, b), suggesting that these actin structures were invadopodias/podosomes. We next investigated whether the NTD or the C-terminal luminal fragment generated by $\mathrm{PC} 7 /$ Furin is/are implicated in 


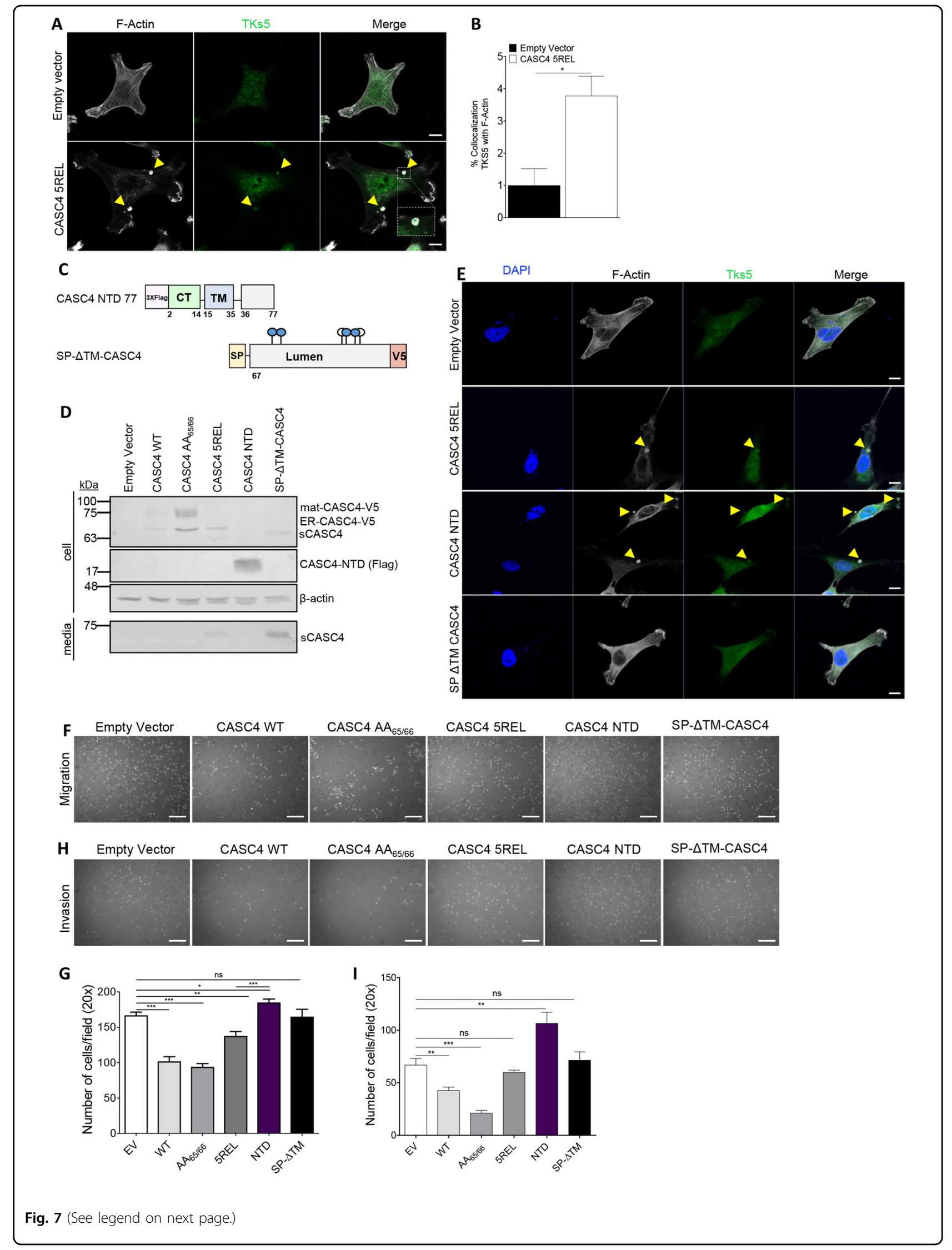


(see figure on previous page)

Fig. 7 CASC4 N-terminal domain (NTD) induces podosome-like structures. $\mathbf{a}$, b Immunofluorescence analysis and quantification of MDA-MB-231 stable cells stained for phalloidin (F-actin; white labeling) or tyrosine kinase substrate 5 (TKS5; green labeling). c Schematic representation of CASC4 truncated mutant (NTD) with a 3xflag in N-terminal and SP- $\mathrm{TM}$-CASC4 with a V5 in C-terminal. The blue circles are depicting potential $\mathrm{N}$-glycosylation sites and the white circles are depicting O-glycosylation sites. $\mathbf{d}$ Western blot analysis of cell lysates and media from MDA-MB-231 cells transiently transfected with different CASC4 constructs. e Immunofluorescence analysis of transiently transfected MDA-MB-231 cells stained for phalloidin (F-actin; white labeling), tyrosine kinase substrate 5 TKS5 (green labeling), and nucleus stained with DAPI (blue labeling). Yellow arrows represent colocalization between F-actin and TKS5. The ability of transiently transfected MDA-MB-231 cells to migrate $(6 \mathrm{~h})(\mathbf{e}, \mathbf{f})$ or invade $(16 \mathrm{~h})(\mathbf{g}, \mathbf{h})$ was assessed by counting the number of cells stained with DAPI on the underside of a polycarbonate membrane under a phase contrast microscope $(\times 20)$.These results are representative of at least three independent experiments. Error bars indicate averaged values \pm standard error from the mean (SEM). P-values: ${ }^{*} P \leq 0.05,{ }^{* *} P<0.01,{ }^{* *} P \leq 0.001$ n.s. not significant (two-sided Student's $t$-test). Scale: $10 \mu \mathrm{m}$.

invadopodias induction. Thus, we generated two constructs, one with a stop codon at 11-residues after the shedding site (aa 1-77, likely processed at $\operatorname{Arg}_{66} \downarrow$ ), to create an artificial NTD fragment, and another secretory protein mimicking the luminal shed domain with an N-terminal PCSK9 signal peptide (SP) ${ }^{43}$ fused to Asn $_{67}$ following the shedding site at $\operatorname{Arg}_{66}$ (SP- $\left.\triangle \mathrm{TM}\right)$ (Fig. 7c). We confirmed the expression of these constructs in MDA-MB-231 cells by WB analyses (Fig. 7d) and immunocytochemistry (Supplementary Fig. S4). We next assessed the presence of the actin rings structures in cells expressing the different constructs by immunofluorescence (Fig. 7e). Cells expressing the 5REL and especially the membrane-bound NTD constructs exhibit the presence of round actin structures colocalizing with Tks5 (yellow arrows). To test the NTD role in inducing invadopodias formation, likely influencing migration/ invasion, we transiently expressed all constructs in MDAMB-231 cells and performed Boyden Invasion and Migration Assays. First, we showed that the double Ala-mutant of the shedding site (CASC4-AA ${ }_{65 / 66}$, Fig. 2c) is 2-fold more active in reducing invasion than the WT-CASC4 (Fig. 7h, i), supporting the protective role of full-length CASC4. In contrast, expression of the NTD significantly enhanced migration/invasion, whereas expression of the SP- $\Delta \mathrm{TM}$ mutant had no effect (Fig. $7 \mathrm{f}-\mathrm{i}$ ). Thus, the N-terminal fragment generated upon shedding of CASC4 is mainly responsible for the cytoskeletal disruption observed in the CASC4-5REL cells.

\section{Discussion}

The PCs have key roles in both health and disease states by cleavage of precursor proteins ${ }^{1}$, which results in the bioactivation of proteins, but sometimes may also generate cleaved products endowed with novel functions ${ }^{44,45}$. While the roles of Furin in proliferative and infectious diseases have been extensively characterized ${ }^{10,46-49}$, those of PC7 are barely defined ${ }^{8,9,12}$. One of the specific functions of PC7 is the shedding of the human type-II TfR $1^{9}$, resulting in the secretion of a circulating sTfR 1 that correlates with iron deficiency ${ }^{50}$. These data and those of the present study suggest that PC7 may shed several typeII transmembrane proteins, and would not represent a rare phenomenon, but rather provide a mechanism to modulate their functions and possibly generate new ones.

A proteomic screen from the media of $\mathrm{HuH} 7$ cells identified three shed type-II transmembrane proteins, TfR1, as well as CASC4 and GPP130. We demonstrated that only PC7 and Furin can shed CASC4 at Arg $66 \downarrow$ and GPP130 (not shown) in post-ER acidic compartments. This CASC4 cleavage generates an N-terminal membrane-bound fragment (NTD; aa 2-65) and a secreted C-terminal fragment starting at $\mathrm{Asn}_{67}$. The only available information on CASC4 is its association with HER2 ${ }^{+}$ overexpression and its differential splicing in breast cancer $^{13,15}$ and glioblastoma ${ }^{16}$. Our METABRIC data indicated that patients with high PCSK7 and high CASC4 had significantly worse prognosis than those with high CASC4 but low PCSK7. In addition, we demonstrated that endogenous shedding of CASC4 is only observed in triplenegative breast cancer cells MDA-MB-231. We thus hypothesized that CASC4 and its shedding are relevant in breast cancer aggressiveness/metastasis. Initial evidence revealed that knockdown of CASC4 mRNA significantly increased cell migration and invasion, suggesting a protective role of CASC4.

To explore the functional consequences of CASC4 shedding, we generated three cells lines expressing either a control empty vector, CASC4-WT or CASC45REL. The data revealed that overexpressed CASC4 enhanced the number of FAs, in part by blunting the activation of the Rho GTPase Cdc42, supporting its effect on the reduced migratory potential of these cells. It is not surprising that Cdc42 activity could be modulated by Golgi-localized CASC4 since Cdc42 localizes to the Golgi apparatus and interacts with regulators of cytoskeleton remodeling and centrosome organization ${ }^{51}$. We further demonstrated that shedding of CASC4 into sCASC4 is a critical event that transforms the protective function of CASC4-WT into a pathogenic one due to the generation of the NTD (Fig. 8). Since Furin/ PC7 shedding likely occurs in the TGN and/or endosomes, CASC4-5REL would remain as full length until it reaches these intracellular destinations, and would partially reduce the levels of active Cdc42 in early Golgi 


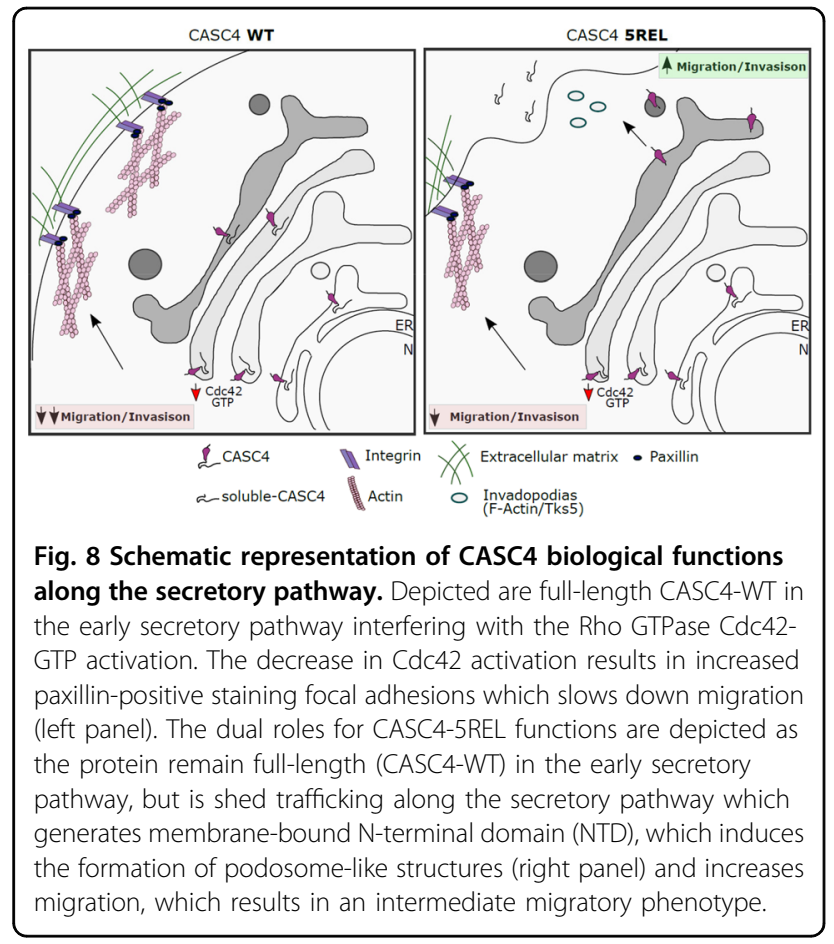

compartments (Fig. 8), like CASC4-WT. This may rationalize the intermediate invasion phenotype observed in cells expressing CASC4-5REL, which when shed later along the secretory pathway would generate an NTD that rather increases podosome formation, known to be associated with enhanced invasion ${ }^{18,19}$. More detailed studies are needed to define the underlying mechanism behind the activity of the NTD in enhancing the formation of podosome-like structures. Since we do not know the physiological function of CASC4, future work will also be needed to investigate the function of the luminal domain of this protein. Similar to other members of the GOLM1 family, CASC4 may act as a co-receptor, as reported for GOLM1/GP73 for the EGF receptor ${ }^{52}$.

The fact that experimental expression of different forms of CASC4 (WT, $\mathrm{AA}_{65 / 66}$ or 5REL) leads to different/ opposing biological consequences in the context of breast cancer, suggests that this protein might have dual actions depending on the associated activity of PC7/Furin. Breast cancer can be categorized pathologically into three main categories: Estrogen/progesterone receptor (ER/PR)-positive, HER2 ${ }^{+}$, or Triple-Negative Breast Cancer (TNBC). Interestingly, in two different clinical breast cancer data sets, METABRIC and TCGA, we found that CASC4 expression positively correlates with the ER/PR status (Supplementary Fig. S5). Correlating the CASC4 mRNA levels to the different molecular subtypes of breast cancer by the PAM50 classification showed similarly that luminal tumors, that are usually ER/PR-positive and have best prognosis, express the highest levels of CASC4
(Supplementary Fig. S5). These evidences strongly pinpoint the significance of exploring the dual roles of CASC4 and its PC7/Furin-shed form in order to better understand the role of CASC4 in different breast cancer subtypes.

Interestingly, analysis of the genes implicated in cancer on chromosome 11, revealed that the locus 11q23.3 is associated with a high incidence of ovarian, breast and uterine cancer (human cancer proteome database http:// canprovar2.zhang-lab.org/chr/chr11.php). Coincidentally, this is the locus of PCSK7 that resides close to the genecluster $A P O A 5 / A P O A 4 / A P O C 3 / A P O A 1$, a region implicated in the regulation of lipoprotein metabolism (Supplementary Fig. S6) $)^{53}$.

In conclusion, our results provide a framework for deciphering the biological functions of CASC4 and more importantly suggest that inhibitors of Furin/PC7 may find clinical applications in breast and ovarian cancers ${ }^{1,5,48}$.

\section{Acknowledgements}

This work was supported by a CIHR Foundation Grant \# 148363 (N.G.S.), a CIHR Project Grant \# MOP-142425 to (J.-F.C.), a Canada Research Chairs in Precursor Proteolysis (N.G.S.; \# 950-231335) and a philanthropic Transat Chair in Breast Cancer Research (J.-F.C.). A.A.T. and I.E.E. are recipients of FRQS Doctoral studentships. S.D. and I.E.E. were also supported by philanthropic studentships from Molson-Bombardier through the IRCM Foundation. We thank Mouna Derbali for cell culture assistance and Brigitte Mary for editorial help.

\section{Author details}

'Laboratory of Biochemical Neuroendocrinology, Montreal Clinical Research Institute (IRCM; Affiliated to the University of Montreal), 110 Pine Ave West, Montreal, QC H2W1R7, Canada. '2aboratory of Cytoskeletal Organization and Cell Migration, Montreal Clinical Research Institute (IRCM; affiliated to the University of Montreal), 110 Pine Ave West, Montreal, QC H2W1R7, Canada. ${ }^{3}$ Ottawa Institute of Systems Biology and Department of Biochemistry, Microbiology and Immunology, Faculty of Medicine, University of Ottawa, Ottawa, ON K1H 8M5, Canada

\section{Conflict of interest}

The authors declare that they have no conflict of interest.

\section{Publisher's note}

Springer Nature remains neutral with regard to jurisdictional claims in published maps and institutional affiliations.

Supplementary Information accompanies this paper at (https://doi.org/ 10.1038/s41419-020-02893-0).

Received: 10 March 2020 Revised: 30 July 2020 Accepted: 31 July 2020 Published online: 20 August 2020

\section{References}

1. Seidah, N. G. \& Prat, A. The biology and therapeutic targeting of the proprotein convertases. Nat. Rev. Drug Discov. 11, 367-383 (2012).

2. Seidah, N. G. \& Chretien, M. Proprotein and prohormone convertases: a family of subtilases generating diverse bioactive polypeptides. Brain Res. 848, 45-62 (1999).

3. Dubois, C. M. et al. Evidence that furin is an authentic transforming growth factor-beta1- converting enzyme. Am. J. Pathol. 158, 305-316 (2001).

4. Yana, I. \& Weiss, S. J. Regulation of membrane type-1 matrix metalloproteinase activation by proprotein convertases. Mol. Biol. Cell 11, 2387-2401 (2000).

5. Bassi, D. E., Fu, J., Lopez de Cicco, R. \& Klein-Szanto, A. J. Proprotein convertases: "master switches" in the regulation of tumor growth and progression. Mol. Carcinog. 44, 151-161 (2005). 
6. Khatib, A. M., Siegfried, G., Chretien, M., Metrakos, P. \& Seidah, N. G. Proprotein convertases in tumor progression and malignancy: novel targets in cancer therapy. Am. J. Pathol. 160, 1921-1935 (2002).

7. Fu, J., Zhang, J., Gong, Y., Testa, C. L. \& Klein-Szanto, A. J. Regulation of HIF-1 alpha by the proprotein convertases furin and PC7 in human squamous carcinoma cells. Mol. Carcinog. 54, 698-706 (2015).

8. Wetsel, W. C. et al. Disruption of the expression of the proprotein convertase PC7 reduces BDNF production and affects learning and memory in mice. Proc. Natl Acad. Sci. USA 110, 17362-17367 (2013).

9. Guillemot, J., Canuel, M., Essalmani, R., Prat, A. \& Seidah, N. G. Implication of the proprotein convertases in iron homeostasis: proprotein convertase 7 sheds human transferrin receptor 1 and furin activates hepcidin. Hepatology 57, 2514-2524 (2013)

10. Thomas, G. Furin at the cutting edge: from protein traffic to embryogenesis and disease. Nat. Rev. Mol. Cell Biol. 3, 753-766 (2002).

11. Ginefra, P., Filippi, B. G. H., Donovan, P., Bessonnard, S. \& Constam, D. B. Compartment-specific biosensors reveal a complementary subcellular distribution of bioactive furin and PC7. Cell Rep. 22, 2176-2189 (2018).

12. Durand, L. et al. The motif EXEXXXL in the cytosolic tail of the secretory human proprotein convertase PC7 regulates its trafficking and cleavage activity. J. Biol. Chem. 295, 2068-2083 (2020).

13. Oh, J. J., Grosshans, D. R., Wong, S. G. \& Slamon, D. J. Identification of differentially expressed genes associated with HER-2/neu overexpression in human breast cancer cells. Nucleic Acids Res. 27, 4008-4017 (1999).

14. Linstedt, A. D., Mehta, A., Suhan, J., Reggio, H. \& Hauri, H. P. Sequence and overexpression of GPP130/GIMPc: evidence for saturable pH-sensitive targeting of a type II early Golgi membrane protein. Mol. Biol. Cell 8, 1073-1087 (1997).

15. Anczukow, O. et al. SRSF1-regulated alternative splicing in breast cancer. Mol. Cell 60, 105-117 (2015).

16. Babenko, V. N. et al. Computer analysis of glioma transcriptome profiling: alternative splicing events. J. Integr. Bioinform 14, 20170022 (2017).

17. Boersema, P. J., Geiger, T., Wisniewski, J. R. \& Mann, M. Quantification of the Nglycosylated secretome by super-SILAC during breast cancer progression and in human blood samples. Mol. Cell. Proteom. 12, 158-171 (2013).

18. Paterson, E. K. \& Courtneidge, S. A. Invadosomes are coming: new insights into function and disease relevance. FEBS J. 285, 8-27 (2018).

19. Seals, D. F. et al. The adaptor protein Tks5/Fish is required for podosome formation and function, and for the protease-driven invasion of cancer cells. Cancer Cell 7, 155-165 (2005).

20. Chen, R., Seebun, D., Ye, M., Zou, H. \& Figeys, D. Site-specific characterization of cell membrane $\mathrm{N}$-glycosylation with integrated hydrophilic interaction chromatography solid phase extraction and LC-MS/MS. J. Proteom. 103, 194-203 (2014).

21. Rajan, S. G. et al. Tracking neural crest cell cycle progression in vivo. Genesis $\mathbf{5 6}$, e23214 (2018)

22. Horzum, U., Ozdil, B. \& Pesen-Okvur, D. Step-by-step quantitative analysis of focal adhesions. MethodsX 1, 56-59 (2014).

23. Curtis, C. et al. The genomic and transcriptomic architecture of 2,000 breast tumours reveals novel subgroups. Nature 486, 346-352 (2012).

24. Pereira, B. et al. The somatic mutation profiles of 2,433 breast cancers refines their genomic and transcriptomic landscapes. Nat. Commun. 7, 11479 (2016).

25. Ciriello, G. et al. Comprehensive molecular portraits of invasive lobular breast cancer. Cell 163, 506-519 (2015).

26. Cerami, E. et al. The cBio cancer genomics portal: an open platform for exploring multidimensional cancer genomics data. Cancer Discov. 2, 401-404 (2012).

27. Gao, J. et al. Integrative analysis of complex cancer genomics and clinical profiles using the cBioPortal. Sci. Signal 6, pl1 (2013).

28. Chen, R., Zou, H. \& Figeys, D. Detergent-assisted glycoprotein capture: a versatile tool for in-depth N-glycoproteome analysis. J. Proteome Res. 15, 2080-2086 (2016).

29. Wong, E., Maretzky, T., Peleg, Y., Blobel, C. P. \& Sagi, I. The Functional maturation of A disintegrin and metalloproteinase (ADAM) 9, 10, and 17 requires processing at a newly identified proprotein convertase (PC) cleavage site. J. Biol. Chem. 290, 12135-12146 (2015).
30. Anderson, E. D., VanSlyke, J. K., Thulin, C. D., Jean, F. \& Thomas, G. Activation of the furin endoprotease is a multiple-step process: requirements for acidification and internal propeptide cleavage. EMBO J. 16, 1508-1518 (1997).

31. Schechter, I. \& Berger, A. On the active site of proteases. 3. Mapping the active site of papain; specific peptide inhibitors of papain. Biochem. Biophys. Res. Commun. 32, 898-902 (1968).

32. Benjannet, S., Rhainds, D., Hamelin, J., Nassoury, N. \& Seidah, N. G. The proprotein convertase PCSK9 is inactivated by furin and/or PC5/6A: functional consequences of natural mutations and post-translational modifications. J. Biol. Chem. 281, 30561-30572 (2006).

33. Krieger, M., Brown, M. S. \& Goldstein, J. L. Isolation of Chinese hamster cell mutants defective in the receptor-mediated endocytosis of low density lipoprotein. J. Mol. Biol. 150, 167-184 (1981).

34. Lippincott-Schwartz, J. et al. Brefeldin A's effects on endosomes, lysosomes, and the TGN suggest a general mechanism for regulating organelle structure and membrane traffic. Cell 67, 601-616 (1991).

35. Aridor, M., Bannykh, S. I., Rowe, T. \& Balch, W. E. Sequential coupling between COPII and COPI vesicle coats in endoplasmic reticulum to Golgi transport. J. Cell Biol. 131, 875-893 (1995).

36. Kim, W. et al. Neuroinflammation-induced interactions between proteaseactivated receptor 1 and proprotein convertases in HIV-associated neurocognitive disorder. Mol. Cell Biol. 35, 3684-3700 (2015).

37. Braun, E. et al. Guanylate-binding proteins 2 and 5 exert broad antiviral activity by inhibiting furin-mediated processing of viral envelope proteins. Cell Rep. 27, 2092-2104 (2019)

38. Nobes, C. D. \& Hall, A. Rho, rac, and cdc42 GTPases regulate the assembly of multimolecular focal complexes associated with actin stress fibers, lamellipodia, and filopodia. Cell 81, 53-62 (1995).

39. Bagci, $H$. et al. Mapping the proximity interaction network of the Rho-family GTPases reveals signalling pathways and regulatory mechanisms. Nat. Cell Biol. 22, 120-134 (2020).

40. Sells, M. A. et al. Human p21-activated kinase (Pak1) regulates actin organization in mammalian cells. Curr. Biol. 7, 202-210 (1997).

41. Manser, E. et al. Expression of constitutively active alpha-PAK reveals effects of the kinase on actin and focal complexes. Mol. Cell. Biol. 17, 1129-1143 (1997).

42. Buccione, R., Orth, J. D. \& McNiven, M. A. Foot and mouth: podosomes, invadopodia and circular dorsal ruffles. Nat. Rev. Mol. Cell Biol. 5, 647-657 (2004).

43. Seidah, N. G. et al. The secretory proprotein convertase neural apoptosisregulated convertase 1 (NARC-1): liver regeneration and neuronal differentiation. Proc. Natl Acad. Sci. USA 100, 928-933 (2003).

44. Seidah, N. G., Sadr, M. S., Chretien, M. \& Mbikay, M. The multifaceted proprotein convertases: their unique, redundant, complementary and opposite functions. J. Biol. Chem. 288, 21473-21481 (2013).

45. Lichtenthaler, S. F., Lemberg, M. K. \& Fluhrer, R. Proteolytic ectodomain shedding of membrane proteins in mammals-hardware, concepts, and recent developments. EMBO J. 37, e99456 (2018).

46. Braun, E. \& Sauter, D. Furin-mediated protein processing in infectious diseases and cancer. Clin. Transl. Immunol. 8, e1073 (2019).

47. Jaaks, P. \& Bernasconi, M. The proprotein convertase furin in tumour progression. Int. J. Cancer 141, 654-663 (2017).

48. Tome, M. et al. Inactivation of proprotein convertases in T cells inhibits PD-1 expression and creates a favorable immune microenvironment in colorectal cancer. Cancer Res. 79, 5008-5021 (2019).

49. Coutard, B. et al. The spike glycoprotein of the new coronavirus 2019-nCoV contains a furin-like cleavage site absent in CoV of the same clade. Antivir. Res. 176, 104742 (2020)

50. Oexle, K. et al. Novel association to the proprotein convertase PCSK7 gene locus revealed by analysing soluble transferrin receptor (sTfR) levels. Hum. Mol Genet. 20, 1042-1047 (2011).

51. Kodani, A., Kristensen, I., Huang, L. \& Sutterlin, C. GM130-dependent control of Cdc42 activity at the Golgi regulates centrosome organization. Mol. Biol. Cell 20, 1192-1200 (2009)

52. Ye, Q. H. et al. GOLM1 modulates EGFR/RTK cell-surface recycling to drive hepatocellular carcinoma metastasis. Cancer Cell 30, 444-458 (2016).

53. Ashraf, $Y$, et al. Proprotein convertase 7 (PCSK7) reduces apoA-V levels. FEBS J. 287, 3565-3578 (2020). 